VOL. $3(1970), 337-348$.

\title{
Interlacing methods and large indecomposables
}

\section{S. E. Dickson and G. M. Kelly}

The method of interlacing of modules, like amalgamation of groups, is a way of getting new objects from old. Briefly, the interlacing module we consider is a certain factor module of a direct sum of copies (finite or infinite) of an original module $M$. The conditions given in a previous paper by the first author in order that the interlacing module (using finitely many copies) be indecomposable are here greatly weakened, and we further allow the number of copies of the original to be infinite. R. Colby has shown that if $R$ is a left artinian ring, the existence of a bound on the number of generators required for any indecomposable finitely-generated left $R$-module implies that $R$ has a distributive lattice of two-sided ideals. This result iș extended to rings whose identity is a sum of orthogonal local idempotents.

For these rings the same distributivity is proved in case every indecomposable interlacing module of the above type which begins with an indecomposable projective $M$ is finitely-generated. A consequence is that any finite-dimensional algebra over a field having infinitely many two-sided ideals has infinite-dimensional indecomposables.

\section{Introduction}

We deal throughout with left modules over a fixed ring $R$, which we shall seldom need to mention explicitly. All our rings have identities

Received 3 August 1970. 
and all our modules are unitary.

Various authors (see in particular Colby [3] and Dickson [5]) have constructed, under suitable hypotheses on $R$, arbitrarily large finitely-generated indecomposable modules, by the use of an interzacing method: one takes a module $M$ containing two isomorphic submodules $S$ and $S^{\prime}$, forms the direct sum of a number of copies of $M$, and identifies the $S$ in the $k$-th copy of $M$ with the $S^{\prime}$ in the $(k+1)-$ th copy; under suitable conditions the resulting quotient module is indecomposable.

Other authors (see Corner [4], Butler [2], Brenner [1]) have shown that, even when $R$ is a finite-dimensional algebra over a field, there may exist infinite-dimensional indecomposable modules. At the moment, it is for rather special classes of such algebras that infinite-dimensional indecomposables have been shown to exist. For some of these classes, however, the indecomposables can have very large infinite dimensions - any cardinal less than the first strongly inaccessible one.

Our aims in the present paper are three: first, to improve as far as we can the interlacing method, weakening the hypotheses under which it produces indecomposables; secondly, to extend it to the countably-infinite case; and thirdly, to prove that a large class of rings admits infinitely-generated indecomposables.

\section{Indecomposability of quotient modules}

Let $i: A \rightarrow B$ be a monomorphism of modules. Wherever we find it convenient, we speak as if $i$ were actually the inclusion of a submodule. In particular, we write the cokernel of $i$ as $p: B \rightarrow B / A$. We write $\operatorname{End} B$ for the ring of endomorphisms of $B$, and $\operatorname{Rad} \operatorname{End} B$ for the Jacobson radical of this. We contemplate the following five conditions that may be satisfied by such a monomorphism $i$ :

Condition I. For every $m: B \rightarrow A, m i=0$.

Condition II. $\operatorname{Ext}^{1}(B, i): \operatorname{Ext}^{1}(B, A) \rightarrow \operatorname{Ext}^{1}(B, B)$ is monomorphic.

Condition III. If $r$ is an idempotent endomorphism of $A$, with $i_{r}=t i$ for some endomorphism $t$ of $B$, then $r=0$ or $r=1$.

Condition IV. If $t$ is an idempotent endomorphism of $B$ with 
$t i=0$ then $t=0$.

Condition V. If $t$ is any endomorphism of $B$ with $t i=0$ then $t \in \operatorname{Rad} \operatorname{End} B$.

2.1. If $A$ is semi-simple, that is if it is the direct sum of a (possibly infinite) number of simple modules, it is easy to see that Condition $I$ is equivalent to the assertion that no non-zero submodule of $A$ is a direct summand of $B$.

2.2. Since we have the exact sequence $0 \rightarrow \operatorname{Hom}(B, A) \rightarrow \operatorname{Hom}(B, B) \rightarrow \operatorname{Hom}(B, B / A) \rightarrow \operatorname{Ext}^{1}(B, A) \rightarrow \operatorname{Ext}^{1}(B, B) \rightarrow \ldots$ it is clear that Condition II is equivalent to the assertion that $\operatorname{Hom}(1, p): \operatorname{Hom}(B, B) \rightarrow \operatorname{Hom}(B, B / A)$ is epimorphic; that is, that every $g: B \rightarrow B / A$ lifts to an $f: B \rightarrow B$ with $p f=g$.

2.3. Condition $\mathrm{V}$ implies Condition IV since if $(1-t) t=0$ and $t \in \operatorname{Rad} \operatorname{End} B$ then $1-t$ is invertible and so $t=0$.

2.4. If $\operatorname{End} B$ is a local ring and if $A \neq 0$, Conditions III and $V$ are automatically satisfied, and so a fortiori is Condition IV. For Condition $V$ this is clear; since $t i=0$ and $i \neq 0, t$ is a non-unit and so belongs to $\operatorname{Rad} \operatorname{End} B$. For Condition III, either $t$ or $1-t$ is a unit; by replacing $r$ by $1-r$ if necessary, we can suppose that $1-t$ is a unit. Since $t i=i r$ we have $\left(t-t^{2}\right) i=i\left(r-r^{2}\right)=0$; for $r$ is idempotent. Since $1-t$ is a unit this gives $t i=0$, so that $i r=0$ and hence $r=0$ since $i$ is monomorphic.

LEMMA 2.5. Let $i$ satisfy Conditions I and II, and let $s$ be an idempotent endomorphism of $B / A$. Then there is an idempotent endomorphism $t$ of $B$ with $p t=s p$.

Proof. By 2.2, Condition II allows us to find $f: B \rightarrow B$ with $p f=s p$. Set $n=f-f^{2}$, and observe that $n$ commutes with $f$. Then $p n=p\left(f-f^{2}\right)=\left(s-s^{2}\right) p=0$, so that $n=i m$ for some $m: B \rightarrow A$. Since $m i=0$ by Condition $I$, it follows that $n^{2}=0$. Setting $t=f-n+2 n f$, and using $f^{2}=f-n$ and $n^{2}=0$, we verify at once that $t^{2}=t$. Since $p n=p i m=0$, we have $p t=p f=s p$.

PROPOSITION 2.6. Let $i$ satisfy Conditions I and II. Then B/A is indecomposable if and only if every idempotent endomorphism of $B$ that 
mops $A$ into $A$ is either 0 or 1 .

Proof. If Let $s$ be an idempotent endomorphism of $B / A$. By Lemma 2.5 there is an idempotent endomorphism $t$ of $B$ with $p t=s p$; thus $t$ maps $A$ into $A$. By hypothesis, $t=0$ or 1 ; since $p$ is epimorphic it follows that $s=0$ or 1 ; thus $B / A$ is indecomposable.

ONLY IF Let $t$ be an idempotent endomorphism of $B$ mapping $A$ into $A$. Then $t$ induces an idempotent endomorphism $s$ of $B / A$ with $s p=p t$. Since $B / A$ is indecomposable, $s=0$ or $1 ;$ on replacing $t$ by $1-t$ if necessary we can suppose that $s=0$. Then since $p t=0$ we have $t=i m$ for some $m: B \rightarrow A$. Since $m i=0$ by Condition $I$, we have $t^{2}=0$; whence $t=0$ since $t=t^{2}$.

COROLLARY 2.7. Let $i$ satisfy Conditions I, II, III, IV. Then $B / A$ is indecomposable.

Proof. Let $t$ be an idempotent endomorphism of $B$ mapping $A$ into $A$. Then $t i=i r$ for some $r: A \rightarrow A$, and $r$ too is idempotent. By Condition III, $r=0$ or $l$; on replacing $t$ by $l-t$ if necessary, we may suppose that $r=0$. Then $t i=0$, so that $t=0$ by Condition IV.

\section{The interlacing construction}

Consider module homomorphisms $\phi, \psi: S+M$; we suppose that $\phi$ (but not necessarily $\psi$ ) is monomorphic. Let $\Omega$ be an ordinal $\geq 1$ that is either a finite ordinal $n$ or else the first infinite ordinal $\omega$. Let $A$ be the direct sum of copies of $S$, indexed by the ordinals $<\Omega$; let $B$ similarly be the direct sum of copies of $M$, indexed by the ordinals $<\Omega$; and define a homomorphism $i: A \rightarrow B$ as follows, in terms of its matrix components $i_{\alpha \beta}$ :

$$
\begin{gathered}
i_{\alpha \alpha}=\phi \text { for all } \alpha, \\
i_{\alpha, \alpha-1}=\psi \text { for all } \alpha \geq 1, \\
i_{\alpha \beta}=0 \text { for all other }(\alpha, \beta) .
\end{gathered}
$$

Thus in the finite case $i$ is the matrix 


$$
i=\left(\begin{array}{llllll}
\phi & 0 & 0 & \ldots & 0 & 0 \\
\psi & \phi & 0 & \ldots & 0 & 0 \\
0 & \psi & \phi & \ldots & 0 & 0 \\
& & \vdots & & & \\
0 & 0 & 0 & & \phi & 0 \\
0 & 0 & 0 & & \psi & \phi
\end{array}\right) .
$$

That $i$ is a monomorphism follows at once from the fact that $\phi$ is a monomorphism. It is our intention to show that $B / A$ is indecomposable under suitably hypotheses on $\phi$ and $\psi$.

We shall need to contemplate for the monomorphism $\phi$ the same Conditions I - V that we contemplated in $\$ 2$ for the monomorphism $i$. We must also contemplate the following two conditions on the ordered pair $\phi, \psi$ :

Condition VI. For any $a, b \in$ End $M$ and any $k \in$ EndS, if $a \psi=b \phi+\phi k$ then $a \psi=0$.

Condition VII. For any $a \in \operatorname{End} M$, if $a \psi=0$ then $a \phi=0$.

LEMMA 3.1. If $\phi, \psi$ satisfy Conditions VI and VII, and if $h: M \rightarrow S$, then $h \phi=h \psi=0$. In particular, $\phi$ satisfies Condition I.

Proof. We have $(\phi h) \psi=\phi(h \psi)$, whence by Condition VI $(\phi h) \psi=0$. It then follows from Condition VII that $(\phi h) \phi=0$. Since $\phi$ is monomorphic, we conclude that $h \psi=h \phi=0$.

PROPOSITION 3.2. Let $\phi$ satisfy Conditions II, III, and $V$, and let $\phi, \psi$ satisfy Conditions VI and VII. Then $i$ satisfies, in the finite case, Conditions I, II, III, and V . In the infinite case, $i$ satisfies Conditions I, II, III, and IV, provided that we add the following extra hypothesis: the functor $\operatorname{Ext}{ }^{l}(M,-)$ preserves countable direct sums.

Proof. $i$ satisfies I: Immediate from Lemma 3.1 .

$i$ satisfies II: Since $\operatorname{Ext}^{1}(B,-)$ is the direct product of the functors $\operatorname{Ext}^{1}(M,-)$, it suffices to prove that $\operatorname{Ext}^{1}(M, i)$ is monomorphic. Now $\operatorname{Ext}^{1}(M, A)$ and $\operatorname{Ext}^{1}(M, B)$ are, using the extra hypothesis in the infinite case, direct sums of copies of $\operatorname{Ext}^{1}(M, S)$ and 
of $\operatorname{Ext}^{1}(M, M)$ respectively; and $\operatorname{Ext}^{1}(M, i)$ is the matrix made from $\operatorname{Ext}^{1}(M, \phi)$ and $\operatorname{Ext}^{1}(M, \psi)$ exactly as the matrix $i$ is made from $\phi$ and $\psi$. That $\operatorname{Ext}^{1}(M, i)$ is monomorphic now follows from the hypothesis that $\operatorname{Ext}^{1}(M, \phi)$ is monomorphic; that is, from Condition II for $\phi$.

$i$ satisfies III: Let $t i=i r$ where $r$ is idempotent. Write $t_{\alpha \beta}$ and $r_{\alpha \beta}(0 \leq \alpha, \beta<\Omega)$ for the matrix components of $t$ and of $r$. For simplicity set $r_{\alpha \beta}=0$ for $\alpha<0$ and (in the finite case) $t_{\alpha \beta}=0$ for $\beta \geq \Omega$. With this convention the equation $t i=i r$ becomes the following set of equations, which hold for all $\alpha, \beta$ with $0 \leq \alpha, \beta<\Omega$ :

$$
t_{\alpha \beta} \phi+t_{\alpha, \beta+1} \psi=\phi r_{\alpha \beta}+\psi r_{\alpha-1, \beta} \text {. }
$$

We first prove by induction on $\alpha$ that $r_{\alpha \beta}=0$ for $\beta>\alpha$; it is true for $\alpha<0$, and we suppose it to be true for all $\alpha^{\prime}<\alpha$. Then by the inductive hypothesis (1) gives

$$
t_{\alpha \beta} \phi+t_{\alpha, \beta+1} \psi=\phi r_{\alpha \beta} \text { for } \beta \geq \alpha \text {, }
$$

whence Condition VI gives

$$
t_{\alpha, \beta+1} \psi=0 \text { for } \beta \geq \alpha
$$

and then Condition VII gives

$$
t_{\alpha, \beta+1} \phi=0 \text { for } \beta \geq \alpha \text {. }
$$

Equations (2), (3), and (4) now give $\phi r_{\alpha \beta}=0$ for $\beta>\alpha$, and since $\phi$ is monomorphic we have the desired result

$$
r_{\alpha \beta}=0 \text { for } \beta>\alpha \text {. }
$$

Since $r^{2}=r$ and since $r_{O B}=0$ for $\beta>0$, we have $r_{00}^{2}=r_{00}$. Moreover (1) gives, using (3), $t_{00} \phi=\phi r_{00}$. It now follows from Condition III for $\phi$ that $r_{00}=0$ or 1 . On replacing $r$ by $1-r$ if necessary, we may suppose that

$$
r_{00}=0 \text {. }
$$

We next prove by induction on $\alpha$ that $r_{\alpha \alpha}=0$. Let $\alpha>0$, and 
suppose it true for all $\alpha^{\prime}<\alpha$. Putting $B=\alpha-1$ in (1) and using the inductive hypothesis gives

$$
t_{\alpha, \alpha-1} \phi+t_{\alpha \alpha} \psi=\phi r_{\alpha, \alpha-1},
$$

whence Condition VI gives

$$
t_{\alpha \alpha} \psi=0
$$

and then Condition VII gives

$$
t_{\alpha \alpha} \phi=0
$$

Putting now $\beta=\alpha$ in ( 1 ), and using ( 8$),(3)$, and (5), we get $\phi r_{\alpha \alpha}=0$, and since $\phi$ is monomorphic we have the desired result

$$
r_{\alpha \alpha}=0
$$

We finally prove by induction on $\alpha-\beta$ that $r_{\alpha \beta}=0$ for $\alpha>\beta$. In view of (5) and (9) the equation $r=r^{2}$ gives, for $\alpha>\beta$,

$$
r_{\alpha \beta}=\sum_{\beta<\gamma<\alpha} r_{\alpha \gamma}^{r} r_{\gamma \beta} .
$$

For $\alpha-\beta=1$, the right side of (10) is vacuous, and we get $r_{\alpha \beta}=0$. Let $\alpha-\beta>1$, and suppose the result is true for all lesser values of $\alpha-\beta$. Then each term on the right side of (10) vanishes by the inductive hypothesis, whence we indeed have

$$
r_{\alpha \beta}=0 \text { for } \alpha>\beta \text {. }
$$

From (5), (9), and (11) we have $r=0$, which completes the proof that $i$ satisfies Condition III.

$i$ satisfies Condition V (finite case): If $t i=0$ equation ( 1 ) gives

$$
t_{\alpha \beta} \phi+t_{\alpha, \beta+1} \psi=0
$$

whence $t_{\alpha \beta} \phi=0$ by Condition VI; and then Condition V for $\phi$ allows us to conclude that $t_{\alpha \beta} \in \operatorname{Rad} \operatorname{EndM}$. As is well known, this is equivalent to the assertion that $t \in \operatorname{Rad} \operatorname{End} B$. 
$i$ satisfies condition IV (infinite case): The above reasoning still gives $t_{\alpha \beta} \in \operatorname{Rad} \operatorname{End} M$, even in the infinite case, but this no longer implies that $t \in \operatorname{Rad} \operatorname{Ena} B$. Thus we cannot get Condition $V$ for $i$ in the infinite case, but the weaker Condition IV will now follow once we prove:

LEMMA 3.3. Let $t$ be an idempotent endomorphism of $B$ with $t_{\alpha \beta} \in \operatorname{Rad} \operatorname{End} M$ for each $\alpha, \beta$. Then $t=0$.

Proof. Write $t_{\beta}$ for the $\beta$-th column of $t$, so that $t_{\beta}$ is the $\beta$-th component homomorphism $t_{\beta}: M \rightarrow B$. It suffices to show that $t_{\beta}=0$ for all $\beta$, and to do this it suffices to show that $t_{\beta} x=0$ for each $x \in M$.

Given $\beta$ and $x$, the element $t_{\beta} x$ of $B$ has components $t_{\alpha \beta} x$, $0 \leq \alpha<\Omega$; and all but a finite number of these components are 0 . Suppose that $t_{\alpha \beta} x=0$ for $\alpha \geq N$. Partition the matrix $t$ and the column-vector $t_{\beta}$ thus:

$$
t=\left(\begin{array}{ll}
a & b \\
c & d
\end{array}\right) \quad \dot{t}_{B}=\left(\begin{array}{l}
p \\
q
\end{array}\right)
$$

where $a$ has $N$ rows and $N$ columns, and $p$ has $N$ entries. Since $(1-t) t=0$ we have $(1-t) t_{B}=0$, or

$$
\left(\begin{array}{cc}
1-a & -b \\
-c & 1-a
\end{array}\right)\left(\begin{array}{l}
p \\
q
\end{array}\right)=0 ;
$$

in particular we have $(1-a) p-b q=0$, and therefore

$$
(1-a) p x=b q x \text {. }
$$

But the assertion that $t_{\alpha \beta^{x}}=0$ for $\alpha \geq N$ is just the assertion that $q x=0 ;$ so we have

$$
(1-a) p x=0
$$

But if $B^{\prime}$ is the direct sum of the first $N$ copies of $M$, we have $a \in \operatorname{Rad} \operatorname{End} B^{\prime}$ since each $t_{\gamma \delta} \in \operatorname{Rad} \operatorname{End} M ;$ hence $1-a$ is invertible, 
and we conclude that $p x=0$. From $p x=0$ and $q x=0$ we now have $t_{\beta} x=0$, as required.

The proof of Proposition 3.2 is now complete. Combining Proposition 3.2 with Corollary 2.7 , and taking account of 2.4 , we have:

THEOREM 3.4. Let $\phi, \psi: S \rightarrow M$ be homomorphisms with $\phi$ monomorphic, and construct $i: A \rightarrow B$ as above. Let $\phi$ satisfy Conditions II, III, and V; in the case when EndM is Zocal and $S \neq 0$, Conditions III and V for $\phi$ are automatic and need not be explicitly imposed. Let $\phi, \psi$ satisfy Conditions VI and VII. In the infinite case, let $\operatorname{Ext}^{1}(M,-)$ preserve countable direct sums. Then $B / A$ is indecomposable.

3.5. In the finite case there is a slightly different interlacing construction we can use. We can take $B$ as before to be the sum of $n$ copies of $M$, but $A$ to be the sum of only $(n-1)$ copies of $S$, with $i$ as the matrix

$$
i=\left(\begin{array}{cccccc}
\phi & 0 & 0 & \ldots & 0 & 0 \\
\psi & \phi & 0 & \ldots & 0 & 0 \\
0 & \psi & \phi & \ldots & 0 & 0 \\
& & \vdots & & & \\
0 & 0 & 0 & \ldots & \psi & \phi \\
0 & 0 & 0 & \ldots & 0 & \psi
\end{array}\right) .
$$

The above arguments all still work with very minor changes, and $B / A$ is again indecomposable under the same conditions.

\section{Rings with large indecomposables}

We call an idempotent $e$ in a ring $R$ a local idempotent, if the ring $e R e$ is local (see Lambek [6], pp. 76-78). We call a ring $R$ special, if 1 admits a decomposition $1=e_{1}+e_{2}+\ldots+e_{m}$ as a sum of orthogonal local idempotents. An artinian ring (one with minimum condition for left ideals) is of course special. Clearly any quotient ring of a special ring is special.

THEOREM 4.1. Let $R$ be a special ring, and suppose that its lattice of two-sided ideals is not distributive. Then $R$ has 
indecomposable modules that are not finitely generated. It also has finitely-generated indecomposable modules requiring an arbitrarily high number of generators.

Proof. Since the lattice of two-sided ideals of $R$ is modular but not distributive, it contains a sublattice of the form

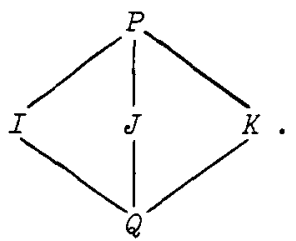

Since an indecomposable $R / Q$-module is also an indecomposable $R$-module, and since $R / Q$ is again special, we may without loss of generality suppose that $Q=0$.

Then given $x \in I$ there is a unique $y \in J$ with $x-y \in K$; write $n x$ for this $y$. Then $\eta: I \rightarrow J$ is an isomorphism of $(R, R)$-bimodules. Let $1=e_{1}+e_{2}+\ldots+e_{m}$ be a representation of the identity of $R$ as a sum of orthogonal local idempotents. Since $I \neq 0$, there must be some $e_{\alpha}$ with $I e_{\alpha} \neq 0$; write $e$ for this $e_{\alpha}$. Then $I e$ and $J e$ are ( $R$, eRe)-bimodules, and $\eta$ restricts to an ( $R$, eRe)-isomorphism $\zeta: I e+J e$.

Take $M$ to be the left $R$-module $R e$. As a direct summand of $R$, $M$ is projective. The endomorphisms of $M$ are exactly the right multiplications by elements of $e$ Re, and $E n d M \cong(e R e)^{O P}$ is therefore local; in particular $M$ is indecomposable.

Take $S$ to be the left $R$-module $I e$. Define $\phi: S \rightarrow M$ to be the inclusion $\mathrm{Ie} \rightarrow \mathrm{Re}$, and $\psi: S \rightarrow M$ to be the isomorphism $\zeta: \mathrm{Ie} \rightarrow J e$ followed by the inclusion $\mathrm{Je} \rightarrow \mathrm{Re}$.

Since $M$ is projective, $\operatorname{Ext}^{1}(M,-)=0$. Thus Condition II for $\phi$ is trivially satisfied, as is the condition that $\operatorname{Ext}^{l}(M,-)$ preserve infinite direct sums. Since $\operatorname{End} M$ is local and $S \neq 0$, Conditions III and $\mathrm{V}$ for $\phi$ are automatically satisfied, by 2.4 .

We have $\operatorname{im} \phi=I e, \operatorname{im} \psi=J e$. Since $I e$ and $J e$ are stable under 
the endomorphisms of $M$, and since $I e \cap \mathrm{Je}=0$, Condition VI is satisfied. Since $I e$ and $J e$ are isomorphic as $(R, e R e)$-bimodules, and hence as $(R$, EndM)-bimodules, Condition VII is satisfied.

If we now carry out the interlacing construction of $\$ 3$, using either the finite or the infinite case, it follows from Theorem 3.4 that $B / A$ is indecomposable.

It remains to prove that $B / A$ is not finitely generated in the infinite case, and requires in the finite case a number of generators that approaches infinity with $n$.

Since $I \cap J=0, I J=0$; and similarly for the other products $I K$ and so on. Since $P=J+K$, it follows that $I P=0 ;$ similarly $J P=0$. Since $P=I+J$, it then follows that $P^{2}=0$. Hence $P$ lies in the radical $N$ of $R$. Since im $\phi$ and im $\psi$ both lie in the submodule $P e$ of $M=R e$, they both lie in $N e$. Thus $B / A$ has a quotient module consisting of the direct sum of $\Omega$ copies of $M / N M$, that is of $R e / N e$. By the theory of local idempotents (see [6], pp. 76-78) $R e / N e$ is a simple left $R$-module, hence $B / A$ cannot be covered by a direct sum of a finite number of copies of $R / N$ (hence of $R$ ) in case $\Omega$ is infinite, and in the finite case an increasingly large number of copies of $R / N$ (hence of $R$ ) is required as $n$ becomes large.

Since a distributive lattice in which all chains have bounded length is finite, we have

COROLLARY 4.2. Let $R$ be an artinian ring whose lattice of two-sided ideals is infinite. Then the conclusions of Theorem 4.1 hold.

4.3. The simplest example to which Theorem 4.1 applies is the $Z_{2}$-algebra $R$ with additive basis $\{1, x, y\}$ and with $x^{2}=y^{2}=x y=y x=0$. The lattice of ideals is

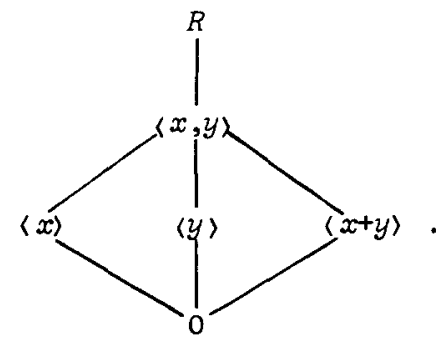


The indecomposable $B / A$ produced by the above process has a basis consisting of elements $u_{\alpha}(0 \leq \alpha<\omega)$ and elements $v_{\alpha}(0 \leq \alpha<\omega)$, with $x u_{\alpha}=u_{\alpha}, y u_{\alpha}=u_{\alpha+1}, x v_{\alpha}=y v_{\alpha}=0$.

\section{References}

[1] Sheila Brenner, "Modular representations of p-groups", J. Algebra 15 (1970), 89-102.

[2] M.C.R. Butler, "On the structure of modules over certain augmented algebras", Proc. London Math. Soc. (tc appear).

[3] R.R. Colby, "On indecomposable modules over rings with minimum condition", Pacific J. Math. 19 (1966), 23-33.

[4] A.L.S. Corner, "Endomorphism algebras of large modules with distinguished submodules", J. Algebra 11 (1969), 155-185.

[5] S.E. Dickson, "On algebras of finite representation type", Trans. Amer. Math. Soc. 135 (1969), 127-141.

[6] J. Lambek, Lectures on rings and modules (Blaisdell, Waltham, Massachussets, 1966).

University of Newcastle,

Newcastle, New South Wales,

and

lowa State University,

Ames, Iowa, USA;

University of New South Wales,

Kensington, New South Wales. 